\title{
CONDITIONS FOR GENERATING A NONVANISHING BOUNDED ANALYTIC FUNCTION
}

\author{
J. H. MANTEL
}

\begin{abstract}
B. A. Taylor and L. A. Rubel have posed the problem of finding necessary and sufficient conditions on a set of given functions $f_{1}, f_{2}, \ldots, f_{n}$ in $H^{\infty}$ such that there exist functions $g_{1}, g_{2}, \ldots, g_{n}$ in $H^{\infty}$ with $\sum_{i=1}^{n} f_{i} g_{i} \neq$ 0 in the open unit disc. L. A. Rubel has conjectured that a necessary and sufficient condition is that there exist a harmonic minorant of $\log \left[\sum_{i=1}^{n}\left|f_{i}\right|\right]$ in the open unit disc. The major result of this paper proves that the conjecture is true if one of the given functions $f_{1}, f_{2}, \ldots, f_{n}$ has a zero set which is an interpolation set for $H^{\infty}$.
\end{abstract}

Let $D$ be the open unit disc in the complex plane and let $H^{\infty}$ denote the space of bounded holomorphic functions on $D$. If $f_{1}, f_{2}, \ldots, f_{n}$ are $n$ given functions in $H^{\infty}$, we seek necessary and sufficient conditions that there are functions $g_{1}, g_{2}, \ldots, g_{n}$ in $H^{\infty}$ with

$$
\sum f_{i}(z) g_{i}(z) \neq 0, \quad z \in D .^{1}
$$

If it happens that $\left|\sum f_{i} g_{i}\right|$ is actually bounded away from zero on $D$, then $f_{1}, f_{2}, \ldots, f_{n}$ generate $H^{\infty}$ (as an ideal); it is a known and difficult theorem of L. Carleson that $f_{1}, f_{2}, \ldots, f_{n}$ generate $H^{\infty}$ if and only if

$$
\sum\left|f_{i}(z)\right| \geqslant \delta>0, \quad z \in D
$$

(see [2, p. 163]). Since we ask here for only the weaker condition that $\Sigma f_{i} g_{i}$ does not vanish in $D$, it is to be expected that (2) will be replaced by some weaker condition. L. A. Rubel has conjectured that (1) holds for some $g_{1}, g_{2}, \ldots, g_{n}$ if and only if the function

$$
\mu(z)=\log \sum\left|f_{i}(z)\right|
$$

has a harmonic minorant on $D .{ }^{2}$ We show in what follows that this conjecture is true under the additional hypothesis that the zero set of some $f_{j}$ is an interpolation sequence for $H^{\infty}$.

A sequence of points $\left\{z_{k}\right\}_{k=1}^{\infty}$ in $D$ is called an interpolation sequence for $H^{\infty}$ if, for each bounded sequence of complex numbers $\left\{w_{k}\right\}_{k=1}^{\infty}$, there exists a function $f$ in $H^{\infty}$ such that $f\left(z_{k}\right)=w_{k}$ for every $k$. A Blaschke product is an

Received by the editors March 10, 1977.

AMS (MOS) subject classifications (1970). Primary 30A76, 30A78, 30A82; Secondary 30A08.

Key words and phrases. Blaschke product, $H^{\infty}$, interpolation sequence for $H^{\infty}$, harmonic minorant, zero set, generating a nonvanishing bounded analytic function.

${ }^{1}$ All summations will be indexed from $i=1$ to $i=n$ unless otherwise indicated.

${ }^{2}$ Private communications. 
analytic function $B$ of the form

$$
B(z)=z^{p} \prod_{k=1}^{\infty}\left[\frac{\bar{\alpha}_{k} \cdot\left(\alpha_{k}-z\right)}{\left|\alpha_{k}\right| \cdot\left(1-\bar{\alpha}_{k} z\right)}\right]^{p_{k}}
$$

where

(i) $p, p_{1}, p_{2}, \ldots$ are nonnegative integers;

(ii) the $\alpha_{k}$ are distinct nonzero numbers in $D$;

(iii) the product $\prod_{k=1}^{\infty}\left|\alpha_{k}\right|^{p_{k}}$ is convergent.

For further discussion on interpolation sequences and Blaschke products, see [2, pp. 66-74, 194-207] and [1, pp. 18-29, 136-143].

Suppose $\Sigma f_{i} g_{i} \neq 0$ in $D$ where the $f_{i}$ 's and $g_{i}$ 's are in $H^{\infty}$. Then $\Sigma f_{i} g_{i}=e^{h}$ where $h$ is analytic in $D$.

Consequently, $e^{\operatorname{Re} h} \leqslant c \Sigma\left|f_{i}\right|$ and so $\operatorname{Re} h-\log c \leqslant \log \Sigma\left|f_{i}\right|$ and $\operatorname{Re} h-$ $\log c$ is harmonic. This shows that the condition that $\log \Sigma\left|f_{i}\right|$ have a harmonic minorant is necessary. It is clearly sufficient for the case $n=1$. For $n \geqslant 2$, suppose the zero set of some $f_{j}$ is finite. If $\mu(z)$ has a harmonic minorant, then the zero sets of $f_{1}, f_{2}, \ldots, f_{n}$ are mutually disjoint. Since the only possible limit points of the zero sets lie on the unit circle, there exists an open set $V$ containing the zero set of $f_{j}$ such that $\left|f_{j}\right| \geqslant \delta_{1}>0$ outside $V$ and $\sum_{k \neq j}\left|f_{k}\right| \geqslant \delta_{2}>0$ on $\bar{V}$. Consequently, $\Sigma\left|f_{i}\right| \geqslant \delta>0$ on the open disc, and by the result of L. Carleson cited above, $f_{1}, f_{2}, \ldots, f_{n}$ generate $H^{\infty}$. Hence we now restrict our attention to the case where $n \geqslant 2$ and the zero sets of the $f_{i}$ 's are all infinite.

THEOREM. Given $f_{1}, f_{2}, \ldots, f_{n}$ in $H^{\infty}$ with the zero set of $f_{1}$ an interpolation sequence for $H^{\infty}$, there exist functions $g_{1}, g_{2}, \ldots, g_{n}$ in $H^{\infty}$ with $\sum f_{i} g_{i} \neq 0$ in $D$ if and only if $\log \Sigma\left|f_{i}\right|$ has a harmonic minorant.

We need a preliminary result:

LEMMA. Let $B$ be a Blaschke product whose zero set $\left\{z_{k}\right\}_{k=1}^{\infty}$ is an interpolation sequence for $H^{\infty}$. Let $f_{1}, f_{2}, \ldots, f_{n}$ be functions in $H^{\infty}$. Then $I_{1}=I_{2}$ where

$$
I_{1}=\left\{B g_{0}+\sum f_{i} g_{i}: g_{0}, g_{1}, g_{2}, \ldots, g_{n} \text { are in } H^{\infty}\right\}
$$

and

$I_{2}=\left\{g \in H^{\infty}:\left|g\left(z_{k}\right)\right|\left(\sum\left|f_{i}\left(z_{k}\right)\right|\right)^{-1} \leqslant M\right.$ for all $k$ where $M$ depends on $\left.g\right\}$.

Proof of Lemma. Let $F=B g_{0}+\sum f_{i} g_{i}$ lie in $I_{1}$. Then

$$
\begin{aligned}
\left|B\left(z_{k}\right) g_{0}\left(z_{k}\right)+\sum f_{i}\left(z_{k}\right) g_{i}\left(z_{k}\right)\right|\left(\sum\left|f_{i}\left(z_{k}\right)\right|\right)^{-1} \\
=\left|\sum f_{i}\left(z_{k}\right) g_{i}\left(z_{k}\right)\right|\left(\sum\left|f_{i}\left(z_{k}\right)\right|\right)^{-1} \leqslant\|g\|_{\infty}
\end{aligned}
$$

for all $k$. Hence $I_{1} \subset I_{2}$. 
Now suppose $g \in I_{2}$. For $i=1,2, \ldots, n$ and $k=1,2,3, \ldots$, define $\alpha_{i k}$ by

$$
\alpha_{i k}= \begin{cases}0 & \text { if } f_{i}\left(z_{k}\right)=0, \\ \operatorname{sgn} f_{i}\left(z_{k}\right) & \text { if } f_{i}\left(z_{k}\right) \neq 0 .\end{cases}
$$

Then $\left\{\alpha_{i k}\right\}_{k=1}^{\infty} \in l^{\infty}$ for $i=1,2, \ldots, n$. Since $\left\{z_{k}\right\}_{k=1}^{\infty}$ is interpolating, there exist $g_{1}, g_{2}, \ldots, g_{n}$ in $H^{\infty}$ such that $g_{i}\left(z_{k}\right)=\alpha_{i k}$ for $i=1,2, \ldots, n$ and $k=1,2,3, \ldots$ Consequently,

$$
\left|g\left(z_{k}\right)\right|\left(\sum f_{i}\left(z_{k}\right) g_{i}\left(z_{k}\right)\right)^{-1}=\left|g\left(z_{k}\right)\right|\left(\sum\left|f_{i}\left(z_{k}\right)\right|\right)^{-1} \leqslant M
$$

for all $k$. So $\left\{g\left(z_{k}\right)\left(\sum f_{i}\left(z_{k}\right) g_{i}\left(z_{k}\right)\right)^{-1}\right\}_{k=1}^{\infty} \in l^{\infty}$, and there exists $h \in H^{\infty}$ such that

$$
h\left(z_{k}\right)=g\left(z_{k}\right)\left(\sum f_{i}\left(z_{k}\right) g_{i}\left(z_{k}\right)\right)^{-1}
$$

for every $k$. We have that $g-h \sum f_{i} g_{i}=0$ on $\left\{z_{k}\right\}_{k=1}^{\infty}$ which implies that $g-h \sum f_{i} g_{i}=B h_{1}$, where $h_{1} \in H^{\infty}$. Thus $g=B h_{1}+\sum f_{i} g_{i} h$ and so $g \in I_{1}$. This shows that $I_{2} \subset I_{1}$, and the proof is complete.

Proof of Theorem. Let $B_{1}$ be the Blaschke factor of $f_{1}$. Suppose $\log \Sigma\left|f_{i}\right|$ has harmonic minorant $\mu$. Let $v$ be a harmonic conjugate of $\mu$. Then $\sum\left|f_{i}\right| \geqslant e^{\mu}=\left|e^{\mu+i v}\right|$ and thus $e^{\mu+i v} \in H^{\infty}$. Let $h=\mu+i v$ and let $\left\{z_{k}\right\}_{k=1}^{\infty}$ be the zero set of $B_{1}$. Then $\left|e^{h\left(z_{k}\right)}\right|\left(\sum\left|f_{i}\left(z_{k}\right)\right|\right)^{-1} \leqslant 1$ for all $k$. By the Lemma, there exist functions $g_{0}, g_{1}, \ldots, g_{n}$ in $H^{\infty}$ such that $B g_{0}+\sum f_{i} g_{i}=e^{h}$. Now $f_{1}=B_{1} G_{1}$, where $G_{1} \in H^{\infty}$ and $G_{1} \neq 0$ in $D$. Hence $B_{1} G_{1} g_{0}+\sum f_{i} g_{i} G_{1}=$ $G_{1} e^{h}$, and the proof is complete.

The condition in the Theorem that $\left\{z_{k}\right\}_{k=1}^{\infty}$ be an interpolation sequence can be weakened so that $\left\{z_{k}\right\}_{k=1}^{\infty}$ is the union of a finite number of interpolation sequences. The argument is by induction on the number of interpolation sequences and is straightforward. P. J. McKenna has announced the following related result: Let $S=\left\{z_{n}\right\}$ be a sequence of points in the open unit disc satisfying the Blaschke condition. Let $\mu$ be the discrete measure concentrated on the sequence $S$, with weights $\mu\left(\left\{z_{n}\right\}\right)=1-\left|z_{n}\right|^{2}$, $n=1,2,3, \ldots$ Then $\mu$ is a Carleson measure if and only if $S$ is a finite union of interpolating sequences.

\section{REFERENCES}

1. P. L. Duren, The theory of $H^{p}$ spaces, Academic Press, New York, 1970.

2. K. Hoffman, Banach spaces of analytic functions, Prentice-Hall, Englewood Cliffs, N. J., 1962.

Unity Mutual Life Insurance Company, 4969 Onondaga Road, Syracuse, New York 13215

Current address: 2111 Cromwell Hills Drive, Cromwell, Connecticut 06416 